\title{
Identification of Substrate-specific Allosteric Fingerprints in Matrix Metalloprotease-1 on Amyloid-beta Peptide Aggregates and Drug Screening With Single Molecule Insights
}

\section{Sumaer Kamboj}

Colorado School of Mines

Chase Harms

Colorado School of Mines

Derek Wright

Colorado School of Mines

Anthony Nash

University of Oxford

Judith Klein-Seetharaman

Colorado School of Mines

Susanta K. Sarkar ( $\square$ ssarkar@mines.edu )

Colorado School of Mines

\section{Research Article}

Keywords: Interactions of MMP1 with A $\beta$-induced aggregates, quantification of allostery, substratespecific allosteric residues

Posted Date: January 27th, 2022

DOI: https://doi.org/10.21203/rs.3.rs-1258797/v1

License: (c) (i) This work is licensed under a Creative Commons Attribution 4.0 International License. Read Full License 


\section{Abstract}

Amyloid-beta peptide $(A \beta)$ is the primary component of water-insoluble extracellular plaques, one of the critical hallmarks of Alzheimer's disease (AD). Matrix metalloproteases (MMPs) are broad-spectrum proteases with diverse functions, including interactions with $A \beta$. Here we report single molecule measurements of MMP1 dynamics on A $\beta$-induced aggregates by calculating Forster Resonance Energy Transfer (FRET) between two dyes attached to the catalytic and hemopexin domains. We show that the two domains of MMP1 prefer closed conformations on A $\beta$-induced aggregates, in contrast to the preference for open conformations on collagen fibril, fibrin, and alpha-synuclein aggregates. We approximated the MMP1 dynamics by a two-state Poisson process and determined the kinetic rates of interconversion between the two states from histograms and correlations of FRET values. We performed molecular docking of MMP1 with A $\beta$ using ClusPro, simulated MMP1 dynamics using different docking poses, and matched the experimental and simulated interdomain dynamics to identify an appropriate pose. We used simulations to create a two-dimensional map of correlations between every pair of MMP1 residues, which shows allosteric communications between the two MMP1 domains. We calculated a Gray Level Co-occurrence Matrix from the two-dimensional map of correlations and quantified MMP1 fluctuations by Shannon entropy. We identified the allosteric residues in the hemopexin domain by identifying residues having strong correlations with the catalytic motif residues. We identified that the residues 1364, G369, P409, G410, and D418 in MMP1 have Aß-specific allosteric correlations with the MMP1 catalytic motif by comparing residues for free and A $\beta$-bound MMP1. We used these $A \beta$-specific allosteric residues to select small molecule ligands after the virtual screening of molecules against $A \beta$ bound MMP1. Molecular understanding of interactions between MMP1 and A $\beta$-induced aggregates and identification of substrate-specific allosteric residues may enable controlling MMP1 function selectively on $A \beta$.

\section{Introduction}

Extracellular amyloid plaques, a key hallmark of Alzheimer's disease (AD) (1), are primarily composed of $A \beta$ (2). Proteolytic cleavage of amyloid precursor protein (APP) a-secretase leads to non-amyloidogenic fragments, neuroprotection, and memory enhancement (3). With age, non-specific fragmentation by $\beta$ secretase and $\gamma$-secretase becomes prevalent, and amyloidogenic fragments, including $A \beta(1-40)$ and $A \beta(1-42)$, appear in the extracellular space (4). Soluble monomers, polymers, and fibrils of extracellular $A \beta$ form the amyloid plaques $(5,6)$.

MMPs are one class of A -degrading enzymes (7). Several MMPs in the 23-member human MMP family (8) interact with $A \beta(9)$ and have relevance in $A D$ and neurodegeneration (10). MMPs are broad-spectrum proteases with diverse proteolytic $(11,12)$ and non-proteolytic intracellular functions $(13)$. MMPs are ubiquitous throughout the human body and are found in extracellular space (14), intracellular space (15), blood (16), intestine (17), and brain (18). The implication of MMPs AD and PD is significant because tetracycline $(19,20)$, an inhibitor of MMPs $(21)$, has shown therapeutic potential in AD (22) and PD (23). In the context of $A D$, an enhanced MMP1 activity correlates with dysfunction of the blood-brain barrier 
(24). MT-MMP1 cleaves $A \beta$ between $\mathrm{H} 14$ and Q15 (25). MMP9 is known to degrade $A \beta$ fibrils in vitro, $A \beta$ plaques in situ, and $A \beta$ in vivo (26-30).

As stated above, degradation of $A \beta$ by MMPs may lead to aggregation. Intriguingly, the broad-spectrum activity of MMPs may also help degrade existing $A \beta$-induced aggregates. However, how water-soluble MMPs interact with water-insoluble $A \beta$-induced aggregates is unclear. Since standard biochemical assays are not suitable for studying water-insoluble substrates, we have developed a single molecule tracking approach and weight-based activity assay. We focused on MMP1, a well-studied MMP of the 23member human MMP family, to study how MMP1 interacts with A $\beta$-induced aggregates at the single molecule level. Since MMP1 interacts with diverse substrates, we need to put the insights into A $-M M P 1$ interactions in the broader context of MMP1 promiscuity for substrate-specific targeting of MMPs in the future. The catalytic domain sequence across the MMP family is very similar, but the hemopexin domain sequence varies $(31,32)$, suggesting a role of communications in substrate diversity and specificity $(12$, 33). Recently, we reported activity-dependent MMP1 dynamics and allosteric communications on type-1 collagen fibrils (34), fibrin (35), and alpha-synuclein-induced aggregates. We found that functionallyrelevant conformations on the three substrates are open MMP1 conformations with the catalytic and hemopexin domains well-separated. These open conformations often accompany a larger catalytic pocket opening of MMP1, facilitating the substrates to get closer to the active site of MMP1. First, however, we need to investigate whether the preference for the open conformations of MMP1 is valid for all substrates and whether allosteric communications are substrate-specific.

This paper reports measurements and analyses of MMP1 interdomain dynamics on water-insoluble A $\beta$ induced aggregates using single molecule FRET (smFRET). We mutated S142 and S366 to CYS for labeling with Alexa555 and Alexa647, a FRET pair of dyes. We calculated the interdomain distance between S142C and S366C from the anticorrelated emission of the two dyes and quantitatively described the dynamics by a two-state Poisson process. A two-state description enables the determination of kinetic rates of interconversion, $\mathrm{k}_{1}$ and $\mathrm{k}_{2}$, between the two states because histograms provide the ratio of $\left(k_{1} / k_{2}\right)$ and correlations of FRET values provide the sum $\left(k_{1}+k_{2}\right)$. We performed MD simulations of MMP1 dynamics and matched them with experimental dynamics. We created a two-dimensional map of fluctuation correlations between every pair of MMP1 residues to show strong allosteric communications in MMP1 and calculated a Gray Level Co-occurrence Matrix to quantify Shannon entropy of the conformational fluctuations. We identified $A \beta$-specific allosteric residues in the hemopexin domain by comparing a normalized correlation greater than 0.8 with the catalytic motif residues for free and $A \beta-$ bound MMP1. Our method of identifying substrate-specific and functionally-relevant allosteric residues may enable modulating one MMP1 function without affecting the other functions, thereby targeting MMPs with fewer side effects.

\section{Results And Discussion}

MMP1 dynamics on A $\beta$-induced aggregates. Using a prism-type Total Internal Reflection Fluorescence (TIRF) microscope, we measured FRET between two dyes attached to the two domains of MMP1 (Figure 
1A). To this end, we created mutants of active (E219) and inactive (Q219) MMP1 with the substitutions S142C and S366C (Figure 1B) for labeling with Alexa555 (donor) and Alexa647 (acceptor). Previously, we showed that labeling does not affect the activity of MMP1. Inactive MMP1 acts as a control to distinguish the effects of enzymatic activity from the effects of the microenvironment and photophysical properties of the dyes. We created a thin layer of water-insoluble $A \beta$-induced aggregates on a quartz slide (Figure 1C). We excited labeled MMPs using a $532 \mathrm{~nm}$ laser inside a flow cell as they interacted with $A \beta$ aggregates (see methods). As MMP1 undergoes interdomain dynamics, the distance and hence, FRET between Alexa555 (donor) and Alexa647 (acceptor) changes, leading to the anticorrelated emission from the two dyes (Figure 1D). When the two MMP1 domains are distant (open conformation), we get low FRET values due to high Alexa555 $\left(I_{D}\right)$ and low Alexa647 $\left(I_{A}\right)$ emissions. In contrast, when the two MMP1 domains are closer (closed conformation), we get high FRET values due to low Alexa555 ( $\left.\mathrm{I}_{\mathrm{D}}\right)$ and high Alexa647 $\left(I_{A}\right)$ emissions. We calculated FRET values using the equation $I_{A} /\left(I_{A}+I_{D}\right)$, where each FRET value determines the distance between the two MMP1 domains. Area-normalized histograms of FRET values without ligands (Figure 1E) suggest that MMP1 prefers closed conformation on $A \beta$ aggregates. This is in contrast to the MMP1's preference for the open conformations on collagen, fibrin, and alpha-synuclein. Although we need more studies in the future to identify the reason for the substrate-dependent preference for the open and closed conformations, substrates play an active role in MMP1 function. A sum of two Gaussians fits the histograms, suggesting an equilibrium of the open and closed conformations. In the presence of tetracycline, a known MMP inhibitor, both active and inactive MMP1 prefer the closed conformations more (Figure 1F). Previously, we argued that tetracycline holds the two MMP1 domains via hydrogen bonds, facilitating the preference for closed conformations. We also calculated the correlation between conformations at different time points. Finally, we fitted both power law and exponential distributions to the autocorrelations and found that an exponential distribution fits the experimental autocorrelations (Figures $\mathbf{1 G}-\mathbf{H}$ ).

A two-state Poisson process description of MMP1 dynamics on Aß-induced aggregates. Recently, we published a quantitative analysis of MMP1 dynamics on collagen fibrils (34) and fibrin (35). A two-state description of MMP1 dynamics allows for easier interpretation of conformational histograms and correlations. The best-fit parameters for the Gaussian fit centers define the two states, S1 (low FRET) and $\mathrm{S} 2$ (high FRET). We calculated the interconversion rates $\mathrm{k} 1(\mathrm{~S} 1 \rightarrow \mathrm{S} 2)$ and $\mathrm{k} 2(\mathrm{~S} 2 \rightarrow \mathrm{S} 1)$ between the two states from the ratio $(k 1 / k 2=$ the area of $S 2$ divided by the area of $S 1)$ and sum $(k 1+k 2=$ the decay rate of autocorrelation).

The two states are $S 1=0.47$ and $S 2=0.52$ on $A \beta$-induced aggregates for active MMP1 without ligand (Table S1). In comparison, the two states are $S 1=0.44$ and $S 2=0.55$ on collagen (34), $S 1=0.42$ and $\mathrm{S} 2=0.51$ on fibrin (35), and S1 $=0.46$ and S2 $=0.52$ on alpha-synuclein aggregates for active MMP1 without ligand. The correlation decay rate is $0.02 \mathrm{~s}^{-1}$ on $A \beta$-induced aggregates for active MMP1 without ligand (Table S1). In comparison, the decay rates are $0.13 \mathrm{~s}^{-1}$ on collagen (34), $0.08 \mathrm{~s}^{-1}$ on fibrin (35), and $0.08 \mathrm{~s}^{-1}$ on $A \beta$ aggregates for active MMP1 without ligand. 
We confirmed the validity of the two-state Poisson process approach to MMP1 dynamics by simulating and analyzing smFRET trajectories using experimentally determined S1, S2, k1, k2, and noise (widths of the histograms). In addition, we recovered the parameters by analyzing the simulated smFRET (Table S2) similar to the analyses of experimental smFRET.

Tetracycline changes the conformational distributions of MMP1 and associated interconversion rates, but the two-state description remains valid (Table S1). Underlying noise can obscure the individual states (Figures 2A-B), but histograms (Figures 2C and 2E) and autocorrelations (Figures 2D and 2F) still recovers the underlying parameters reasonably well (Table S2). The presence of noise increases the width of conformational histograms but subtly affects autocorrelations. In the absence of noise, both powerlaw and exponential distributions fit the autocorrelations (Figure 2F). However, with noise, the y-intercept of the autocorrelations decreases, and only an exponential distribution fits the autocorrelations. The decay rate of autocorrelations does not change as the noise level varies, and an exponential fit recovers the underlying decay rate with and without noise. In other words, the presence of noise can convert a power law correlation into an exponential correlation, similar to the conversion of a Lorentzian line shape into a Gaussian line shape by noise (36).

MMP1 dynamics depend on the MMP1-A $\beta$ binding pose. Since crystal structures of A $\beta$-bound MMP1 do not exist, we predicted the binding poses of A (PDB ID 1IYT) and MMP1 (PDB ID 4AUO) using molecular docking software ClusPro $(37,38)$. To mimic experiments, we selected the three best scoring poses (Figures $3 \mathrm{~A}-\mathrm{C}$ ) and performed all-atom MD simulations at $22^{\circ} \mathrm{C}$ for active and inactive MMP1. Since the computational binding energy may not be the best indicator of appropriate docking poses (39), we leveraged known cleavage sites for a similar MMP. Membrane tethered MT-MMP1, a collagenase similar to MMP1, cleaves $A \beta$ and produces several fragments, including $A \beta$ fragments with amino acids $D 1-H 14$, D1-Q15, D1-L17, V18-V36, and V12-A30 (25). These cleavage sites are located around the middle of A closer to the MMP1 catalytic site in pose 3 (Figure 3C). Figures 3D-F show the distributions of S142-S366 distance, which show that active MMP1 prefers closed conformations (high FRET) more than inactive MMP1, in agreement with experiments (Figure 1E). However, simulated distribution for pose 3 (Figure 3F) leads to a better match of the relative heights of the two peaks with the experimental distribution (Figure 1E). Based on this match between experiments and simulations, we considered pose 3 a relevant binding pose between MMP1 and $A \beta$ for further analysis.

MMP1 catalytic pocket opening shows more peaks (Figures 3G-I). Since a larger catalytic pocket opening may enable substrates to get closer to the active site, we checked for correlations between the interdomain separation and catalytic pocket opening. The correlations between the catalytic pocket opening and interdomain separation are positive for active MMP1 and negative for inactive MMP1 for pose 1 and pose 3 (Figures $3 \mathbf{J}$ and $3 \mathrm{~L}$ ). This is consistent with previous reports of a positive correlation between the catalytic pocket opening and interdomain separation for MMP1 dynamics on collagen (34) and fibrin (35). A positive correlation between the distances further suggests that pose 3 may be a functional binding pose. 
In summary, a combination of known cleavage sites, single molecule measurements of dynamics, and all-atom simulations suggest that pose 3 may be a functionally relevant binding pose. However, there are some caveats because we performed experiments on $A \beta$ aggregates and performed simulations on $A \beta$ monomers. We took a similar approach for collagen and found that simulations on collagen monomer agreed with experiments on collagen fibril when we restrained the collagen backbone (34), suggesting strains in collagen monomers inside collagen fibrils. For $A \beta$, we did not have to restrain the $A \beta$ backbone, suggesting a lack of strain in $A \beta$ aggregates. Nevertheless, the two-way feedback between experiments and simulations provides a starting point for a molecular-level understanding of MMP1-A $\beta$ interactions.

Quantification of MMP1 dynamics on A $\beta$ reveals allostery. We quantified dynamics using correlations because a correlated motion suggests a decrease in randomness or lower entropy (Figure 4). We divided all-atom simulations into $1 \mathrm{~ns}$ long windows, determined the radial coordinates for the alpha carbon in each residue, calculated correlations of fluctuations (Equation 2 in methods), and normalized correlations to a range of 0 to 1 by subtracting the minimum and then dividing by the maximum. Figure 4A shows correlations of simulated interdomain separation, consistent with correlations in experimental dynamics (Figure 1G).

We calculated correlations between each pair of residues to check for allosteric communications. Figures 4B-C show the matrix of correlation values at lag number 1, averaged over $20 \mathrm{~ns}$ (see supplementary information for the meaning of lag numbers and correlation calculations). The catalytic domain residues (F100-Y260) have strong correlations with the hemopexin domain residues (D279-C466), suggesting allosteric communications in MMP1 (Figures 4B-C). We used Shannon entropy to quantify randomness in MMP1 dynamics. To this end, we divided the correlation values to create ten bins of width 0.1 , calculated $10 \times 10$ gray-level co-occurrence matrix (GLCM), and defined Shannon entropy $S=-\sum p_{i} \ln p_{i}$, where $p_{i}$ is the probability of a microstate(see supplementary information for an explanation of Shannon entropy calculations). The time-evolutions of Shannon entropy for pose 3 are shown in Figure 4D.

\section{Changes at the catalytic site and identification of allosteric residues in MMP1. We compared two} dimensional correlation plots of $A \beta$-bound active MMP1 (Figure 5A) and A 5B) at $37^{\circ} \mathrm{C}$. We considered the catalytic residue E219 as the origin and plotted the pairwise distance between E219 and each of the catalytic motif residues HELGHSLGLSH (eleven residues from 218 to 228) in three dimensions (Figure 5C).

The symbol size at each location is proportional to the standard deviation of the pairwise distance. The comparison reveals that the configuration at the MMP1 catalytic site differs considerably between active and inactive MMP1. Figure 5D shows the distributions of correlation values in Figures 5A-B. We used the threshold correlation values at 0.8 , the peak probability density $(\sim 2)$ divided by e $(\sim 2.7)$. We found all the residues with normalized correlations greater than 0.8 in Figure $5 \mathrm{~A}$ and Figure 5B with the catalytic motif residues HELGHSLGLSH. Comparing the residues between Aß-bound active MMP1 with free active MMP1, we identified that the residues $1364, \mathrm{G} 369, \mathrm{P} 409, \mathrm{G} 410$, and D418 are common among the three repeats of $A \beta$-bound active MMP1 simulations and have exclusive correlations for $A \beta$-bound active 
MMP1. Identifying substrate-specific allosteric residues is significant because it may enable controlling one MMP1 function without affecting the other functions.

\section{Substrate-specific allosteric residues in MMP1 enable single-molecule insights into selecting lead} molecules. As a proof of principle, we wanted to check if $A \beta$-specific allosteric residues may help the selection of drugs because $A \beta$ has its unique signature or "fingerprint" on MMP1 at the catalytic and distant allosteric sites. To this end, we screened 9000 FDA-approved drugs (see methods) from the ZINC15 database and selected compounds for which the difference between the binding affinities at the top two binding modes is at least $10 \%$ because we want to find drugs that bind preferably to a specific site on MMP1. With a 10\% difference, we found 600 drugs out of 9000 starting drugs. Next, we considered the top affinity site for each compound, identified the MMP1 residue closest to each drug for each pose (Figure $6 \mathrm{~A}-\mathrm{C}$ ). Then, we further selected drugs by identifying drugs binding within \pm 2 residues around the A $\beta$-specific allosteric residues (I364, G369, P409, G410, and D418) to only 23 lead molecules. Figures $6 \mathrm{D}$ shows a few drugs with their predicted affinities near allosteric residues in pose 3 . Figure $6 \mathrm{E}$ shows how Trosec, the drug with the highest predicted affinity, binds to residue K362. These results suggest that we may be able to identify drugs with an exclusive binding preference for an allosteric site on MMP1 by screening a more significant number of compounds, potentially reducing off-target effects. Since MMPs interact with and degrade many biomolecules in the human body, substrate-specific allosteric residues or "allosteric fingerprints" may alter one MMP1 function without affecting its other activities using allosteric ligands.

\section{Conclusions}

In summary, we measured and quantified the interdomain dynamics of MMP1 on A $\beta$ aggregates at the single molecule level. We modeled the dynamics as a two-state Poisson process and determined the two states' interconversion rates. Distributions of conformations for active and inactive MMP1 suggest that MMP1 prefers closed conformations on A $\beta$ aggregates. In contrast, we previously reported that MMP1 prefers open conformations on collagen (34), fibrin (35), and alpha-synuclein aggregates. Since there is no crystal structure for $A \beta$-bound MMP1, we predicted the binding poses using molecular docking. We performed all-atom simulations of dynamics for different binding poses between A $\beta$ and MMP1, calculated histograms of interdomain dynamics from simulations, and compared them with single molecule measurements. A comparison between experiments and simulations suggest that MMP1 binds between residues $\mathrm{V} 12$ and $L 17$ of $A \beta$ (pose 3 ).

There are caveats because we performed simulations on $A \beta$ monomer and experiments on aggregates. Nevertheless, any degree of agreement between experiments and simulations provides a starting point for integrative analysis. We took a similar approach for collagen and found that simulations of MMP1 dynamics on a collagen monomer agreed with experiments on collagen fibril when the collagen backbone was restrained (34), suggesting the presence of strains in collagen monomers inside collagen fibrils. For $A \beta$, we did not have to restrain the $A \beta$ backbone for agreement between simulations and experiments, suggesting a lack of strain in $A \beta$ aggregates. 
We used simulations informed by experiments to quantify the randomness of dynamics by calculating correlations between each pair of residues in MMP1 and creating a 2D map, which showed strong allostery between the two MMP1 domains. A comparison of 2D correlation matrices for free and A $\beta$ bound MMP1 identified changes at the catalytic motif and Aß-specific allosteric residues in MMP1. We also quantified randomness by defining a GLCM matrix to calculate Shannon entropy at each time point.

We performed virtual screening of approximately 9000 FDA-approved molecules against three predicted binding poses between $A \beta$ and MMP1 and compared the screening results with those for free MMP1. We found that virtual screening results change upon binding $A \beta$ and depend on the binding pose. Substrateand pose-dependent screening results suggest that we need to consider the substrate, the binding pose, and the ligand-binding site targeting MMP1. The integrative approach combining experiments and simulations enables the identification of substrate-dependent allostery at the single molecule level. Our results may pave the way for substrate-specific control of MMP activity using allosteric ligands for fewer side effects.

\section{Materials And Methods}

Purification of MMP1 and A $\boldsymbol{\beta}$. We inserted MMP1 and A $\beta$ sequences, optimized for expression in $E$. coli, into the pET21b+ vector between Ndel (N-terminal) and HindIII (C-terminal) restriction sites. We transformed the plasmids into Rosetta (DE3) pLysS E. coli (Millipore, Cat\# 70956-4) for protein expression. We purified MMP1 and $A \beta$, as described in our previous publications $(40,41)$. The method of purifying $A \beta$ also produced $A \beta$-induced aggregates used in experiments.

Single molecule measurements of MMP1 dynamics. For smFRET measurements of MMP1 interdomain dynamics, we used active and catalytically inactive mutant (E219Q) of MMP1. We introduced two mutations, S142C and S366C, to facilitate labeling with Alexa555 and Alexa647 dyes. We created a thin layer of $A \beta$ aggregates on a quartz slide and made a flow cell for single molecule experiments using double-sided adhesive tape sandwiched between the quartz slide and a glass coverslip. Labeled MMPs were flowed into the flow cell and excited at $532 \mathrm{~nm}$ wavelength. We used the evanescent wave created at the quartz slide-solution interface in a TIRF microscope to reduce background noise. We imaged emissions from Alexa555 and Alexa647 to detect relative motion between the two MMP1 domains. Any interdomain motion would lead to a non-radiative transfer of energy between the two dyes due to FRET, increasing the emission from Alexa647 $\left(I_{A}\right)$ and simultaneously decreasing the emission from Alexa555 $\left(I_{D}\right)$. We calculated approximate FRET efficiency by $I_{A} /\left(I_{A}+I_{D}\right)(42)$. Our previous publications have described more details about single-molecule experiments and analyses (43-46).

All-atom simulations. MD simulations of dynamics involve four steps: (a) construction of structures using available PDB files, (b) preparatory steps before production simulations, (c) production simulations of dynamics, and (d) analyses of simulated dynamics. 
Model construction of free and A 3 -bound MMP1. We created PDB files for MD simulations from PDB ID 4AUO for MMP1 (47) and PDB ID 1IYT for $A \beta$ (48). To predict binding poses between MMP1 and A $\beta$, we used the ClusPro web server (https://cluspro.bu.edu/) with default parameters $(37,38)$ and submitted PDB ID 1IYT as the receptor and only MMP1 part from PDB ID 4AUO as the ligand. We selected the three best scoring binding poses between MMP1 and $A \beta$.

We replaced A219 with E219 (active) and Q219 (inactive), using PyMOL's mutagenesis function (PyMOL Molecular Graphics System, Version 2.0). E219 (40) is the same residue as E200 (47), which differs due to the first amino acid used for counting. Full-length MMP1 has 469 residues. The first six amino acids are MHSFPP, and the last six amino acids are FNCRKN. For reference, PDB ID 4AUO starts with FVLTEG (47) and has 366 residues. We counted from MHSFPP, and as such, FVLTEG numbers are 100, 101, 102, 103,104 , and 106. The catalytic triad HEL residues are H218, E219, and L220.

We replaced the missing side-chain atoms in the PDB files using Chimera's rotamer tool (49). We edited the PDB files and removed zinc ions, calcium ions, and water molecules. We used the resulting PDB files to perform molecular dynamics simulations, as described below.

Setting up MD simulations. We performed MD simulations using Gromacs 2021.2 software package (50) by following the steps of a tutorial (http://www.mdtutorials.com/gmx/) (51). We used the Amber99 force field (52) and SPCE water model (53). We configured the parameters such that the protein structures were placed at the center of a cubic box with 3D period boundary conditions and solvated with water. Then, we added ions to neutralize the charge of the overall system. We then used the steepest descent algorithm to minimize the system's energy and equilibrated the system by NVT and NPT ensemble simulations. First, we performed the NVT simulation and set the mean temperature at the desired temperature of $295 \mathrm{~K}$ ( 22 $\left.{ }^{\circ} \mathrm{C}\right)$ or $310 \mathrm{~K}\left(37^{\circ} \mathrm{C}\right)$ using a Berendsen thermostat for $100 \mathrm{ps}$. We used the Verlet cut-off scheme for neighbor searching and updated the neighbor list every $20 \mathrm{fs}$. We used the particle mesh Ewald scheme to calculate the electrostatic interactions with a cubic interpolation order of 4 and a cut-off at $1 \mathrm{~nm}$. We assigned initial velocities using a Maxwell distribution from the corresponding temperature. In the NPT simulation, we maintained velocities from the NVT simulation output, and the pressure was set to 1 bar using a Parrinello-Rahman barostat for an additional 100 ps.

Production MD simulations. Once the NVT and NPT simulations were finished and the system equilibrated, we removed the position restraints and ran the production MD simulations. Each simulation ran $20 \mathrm{~ns}$ long, simulated at $2 \mathrm{fs} / \mathrm{step}$, and sampled every $5 \mathrm{ps}$. We repeated simulations three times for each condition. We edited the final coordinates in the trajectory file to correct for periodicity and center the protein complex. The distances between the catalytic pocket residues and the serine residues (interdomain distance) are then measured at every time step using the Gromacs distance function. We measured the interdomain separation between the alpha carbons of residues S142 and S366 and the catalytic pocket opening between the alpha carbons of residues N171 and T230. To calculate the correlation and Shannon entropy, we recorded the alpha carbon's coordinates in each residue of MMP1. 
Analyses of experimental and simulated dynamics. We quantified dynamics by conformational histograms and correlations, as we did for previous publications $(34,35)$. For histograms, we chose bin widths equal to or larger than $1 / \sqrt{N}$, where $N$ is the number of data points. We calculated the error in each bin as the square root of the bin count. To obtain the probability density function for conformations, we divided the bin counts and errors by the histogram area so that the area under the normalized histogram equals 1 . We fitted a sum of two Gaussians to the area-normalized histograms to determine the two primary conformational states of MMP1 and their widths:

$$
y=a_{1} \times e^{-\frac{\left(x-b_{1}\right)^{2}}{c_{1}^{2}}}+a_{2} \times e^{-\frac{\left(x-b_{2}\right)^{2}}{c_{2}^{2}}}
$$

1

where a's, b's, and c's are amplitudes, centers, and widths of the Gaussians. Two Gaussian centers, b1 and b2, are the two states, S1 and S2, of MMP1. The widths of the Gaussians are fluctuations around the two states that we used for Poisson process simulations and analyses.

For correlations among conformations, we used the following equation:

$$
C(\tau)=\frac{1}{N-\tau} \sum_{t=1}^{N-\tau}\left\{I(t)-\frac{1}{N-\tau} \sum_{t^{\prime}=1}^{N-\tau} I\left(t^{\prime}\right)\right\} \times\left\{I(t+\tau)-\frac{1}{N-\tau} \sum_{t^{\prime}=1+\tau}^{N} I\left(t^{\prime}\right)\right\}
$$

2

where $C(\tau)$ is the correlation at lag number $\tau, N$ is the number of points in a FRET time series, and $I(t)$ is the FRET value at $t$. For autocorrelations, both factors in curly brackets were from the same time series. For crosscorrelations, the two factors in curly brackets were from different time series representing coordinates of different residues.

We normalized correlations by dividing correlation values at each lag by the correlation value at $\tau=0$. We fitted correlations between $\tau=1$ and $\tau=1000$ to both power-law and exponential distributions. For power law, we used a form of Pareto distribution (54) that satisfies the boundary conditions, i.e., $C(\tau=0)=1$ at $t=0$ and $C(\tau=\infty)=0$ at $t=\infty$. Specifically, we fitted the following equations to correlation values:

$$
\begin{gathered}
C(\tau)=(a \times \tau+1)^{-b} \\
C(\tau)=d \times \exp ^{-e \times \tau}+f
\end{gathered}
$$

3

Also, we used linear regression analysis to quantify correlations between the catalytic pocket opening and interdomain separation from simulations. We used the interdomain distance as the single predictor variable and the catalytic pocket distance as the response variable. We used the following equation for linear regression analysis: 


$$
y_{i}=b_{0}+b_{1} \times x_{i}
$$

where $b_{0}$ and $b_{1}$ are the estimated fit parameters. We calculated the error of each predictor value as the $95 \%$ confidence interval around the mean.

Single molecule insights into the virtual screening. We used PDB files for free MMP1 and three binding poses between $A \beta$ and MMP1 as the receptors. All PDB files were derived from PDB ID 4AUO. We opened PDB files in AutoDockTools, added polar hydrogens, and created a .pdbqt file. We selected 9000 FDAapproved molecules from the ZINC15 database and downloaded them as an sdf file for ligands. We used OpenBabel to convert the sdf file into a pdbqt file. Then, we used AutoDock Vina to create individual .pdbqt files using vina_split on a Linux terminal. We also created a txt file containing filenames for the selected molecules and a configuration file to specify the parameters of screening in AutoDock Vina. The configuration file is a txt file with receptors' filename, docking box coordinates, number of modes, energy range, and exhaustiveness. We used the Graphical User Interface in AutoDockTools to obtain the coordinates by opening the Grid menu and selecting Grid Box. We adjusted the box size to enclose the whole MMP1 to identify each compound's binding locations and affinities using an exhaustiveness of 10 for quicker screening. After the virtual screening, we obtained a pdbqt file of the top 10 docking modes and a log of their binding affinity scores. We created scatter plots in Matlab to compare virtual screening against three binding poses of A $\beta$-bound MMP1 with screening against free MMP1. We identified molecules binding near the $A \beta$-specific allosteric residues in MMP1.

\section{Declarations}

\section{Acknowledgments}

One grant to S.K.S. and J.K.S. from the National Institutes of Health (RGM137295A) partially supported this work. A.N. is funded by the NIHR Oxford Health NHS Foundation Trust Biomedical Research Centre. This work was partially supported by the computational resources provided by Science Outreach Servers (www.scienceoutreachservers.org).

\section{Author contributions}

S.K.S. conceived and designed the overall project, S.K. performed experiments, C.H., A.N., and D.W. performed all-atom simulations and virtual screening, D.W. performed Shannon entropy calculations, S.K., C.H., D.W., A.N., J.K.S., and S.K.S. analyzed data. S.K.S. wrote the manuscript. All authors edited the manuscript.

\section{Competing financial interests}

The authors declare no competing financial interests.

\section{Data availability}


All data generated or analyzed during this study are included in this published article and its supplementary information files.

\section{References}

1. Haass, C., and D. J. Selkoe. 2007. Soluble protein oligomers in neurodegeneration: lessons from the Alzheimer's amyloid $\beta$-peptide. Nature reviews Molecular cell biology 8(2):101-112.

2. De Strooper, B. 2010. Proteases and proteolysis in Alzheimer disease: a multifactorial view on the disease process. Physiological reviews 90(2):465-494.

3. Ghiso, J., and B. Frangione. 2002. Amyloidosis and Alzheimer's disease. Advanced drug delivery reviews 54(12):1539-1551.

4. Chen, M. 2015. The maze of APP processing in Alzheimer's disease: where did we go wrong in reasoning? Frontiers in Cellular Neuroscience 9:186.

5. Pryor, N. E., M. A. Moss, and C. N. Hestekin. 2012. Unraveling the early events of amyloid- $\beta$ protein $(A B)$ aggregation: techniques for the determination of $A \beta$ aggregate size. International Journal of Molecular Sciences 13(3):3038-3072.

6. Hamley, I. W. 2012. The amyloid beta peptide: a chemist's perspective. Role in Alzheimer's and fibrillization. Chemical reviews 112(10):5147-5192.

7. Saido, T., and M. A. Leissring. 2012. Proteolytic degradation of amyloid $\beta$-protein. Cold Spring Harbor perspectives in medicine 2(6):a006379.

8. Tallant, C., A. Marrero, and F. X. Gomis-Rüth. 2010. Matrix metalloproteinases: fold and function of their catalytic domains. Biochimica et Biophysica Acta (BBA)-Molecular Cell Research 1803(1):20-28.

9. Wang, X.-X., M.-S. Tan, J.-T. Yu, and L. Tan. 2014. Matrix metalloproteinases and their multiple roles in Alzheimer's disease. BioMed research international 2014.

10. Mroczko, B., M. Groblewska, and M. Barcikowska. 2013. The role of matrix metalloproteinases and tissue inhibitors of metalloproteinases in the pathophysiology of neurodegeneration: a literature study. Journal of Alzheimer's Disease 37(2):273-283.

11. Rodríguez, D., C. J. Morrison, and C. M. Overall. 2010. Matrix metalloproteinases: what do they not do? New substrates and biological roles identified by murine models and proteomics. Biochimica et Biophysica Acta (BBA)-Molecular Cell Research 1803(1):39-54.

12. Morrison, C. J., G. S. Butler, D. Rodríguez, and C. M. Overall. 2009. Matrix metalloproteinase proteomics: substrates, targets, and therapy. Current opinion in cell biology 21(5):645-653.

13. Jobin, P. G., G. S. Butler, and C. M. Overall. 2017. New intracellular activities of matrix metalloproteinases shine in the moonlight. Biochimica et Biophysica Acta (BBA)-Molecular Cell Research 1864(11):2043-2055.

14. Lukes, A., S. Mun-Bryce, M. Lukes, and G. A. Rosenberg. 1999. Extracellular matrix degradation by metalloproteinases and central nervous system diseases. Molecular neurobiology 19(3):267-284. 
15. Cauwe, B., and G. Opdenakker. 2010. Intracellular substrate cleavage: a novel dimension in the biochemistry, biology and pathology of matrix metalloproteinases. Critical reviews in biochemistry and molecular biology 45(5):351-423.

16. Zucker, S., M. Hymowitz, C. Conner, H. M. Zarrabi, A. N. Hurewitz, L. Matrisian, D. Boyd, G. Nicolson, and S. Montana. 1999. Measurement of matrix metalloproteinases and tissue inhibitors of metalloproteinases in blood and tissues: clinical and experimental applications. Annals of the New York Academy of Sciences 878(1):212-227.

17. Schuppan, D., and E. Hahn. 2000. MMPs in the gut: inflammation hits the matrix. Gut 47(1):12-14.

18. Dzwonek, J., M. Rylski, and L. Kaczmarek. 2004. Matrix metalloproteinases and their endogenous inhibitors in neuronal physiology of the adult brain. FEBS letters 567(1):129-135.

19. Greenwald, R., L. Golub, N. Ramamurthy, M. Chowdhury, S. Moak, and T. Sorsa. 1998. In vitro sensitivity of the three mammalian collagenases to tetracycline inhibition: relationship to bone and cartilage degradation. Bone 22(1):33-38.

20. Federici, T. J. 2011. The non-antibiotic properties of tetracyclines: clinical potential in ophthalmic disease. Pharmacological research 64(6):614-623.

21. Acharya, M. R., J. Venitz, W. D. Figg, and A. Sparreboom. 2004. Chemically modified tetracyclines as inhibitors of matrix metalloproteinases. Drug Resistance Updates 7(3):195-208.

22. Balducci, C., and G. Forloni. 2019. Doxycycline for Alzheimer's disease: fighting $\beta$-amyloid oligomers and neuroinflammation. Frontiers in pharmacology 10.

23. Bortolanza, M., G. C. Nascimento, S. B. Socias, D. Ploper, R. N. Chehín, R. Raisman-Vozari, and E. DelBel. 2018. Tetracycline repurposing in neurodegeneration: focus on Parkinson's disease. Journal of Neural Transmission 125(10):1403-1415.

24. Lorenzl, S., K. Buerger, H. Hampel, and M. F. Beal. 2008. Profiles of matrix metalloproteinases and their inhibitors in plasma of patients with dementia. International psychogeriatrics 20(1):67.

25. Liao, M.-C., and W. E. Van Nostrand. 2010. Degradation of soluble and fibrillar amyloid $\beta$-protein by matrix metalloproteinase (MT1-MMP) in vitro. Biochemistry 49(6):1127-1136.

26. Backstrom, J. R., G. P. Lim, M. J. Cullen, and Z. A. Tökés. 1996. Matrix metalloproteinase-9 (MMP-9) is synthesized in neurons of the human hippocampus and is capable of degrading the amyloid- $\beta$ peptide (1-40). Journal of Neuroscience 16(24):7910-7919.

27. Yan, P., X. Hu, H. Song, K. Yin, R. J. Bateman, J. R. Cirrito, Q. Xiao, F. F. Hsu, J. W. Turk, and J. Xu. 2006. Matrix metalloproteinase-9 degrades amyloid- $\beta$ fibrils in vitro and compact plaques in situ. Journal of Biological Chemistry 281(34):24566-24574.

28. Li, Z., A. Chalazonitis, Y.-y. Huang, J. J. Mann, K. G. Margolis, Q. M. Yang, D. O. Kim, F. Côté, J. Mallet, and M. D. Gershon. 2011. Essential roles of enteric neuronal serotonin in gastrointestinal motility and the development/survival of enteric dopaminergic neurons. Journal of Neuroscience 31(24):89989009.

29. Hernandez-Guillamon, M., S. Mawhirt, S. Blais, J. Montaner, T. A. Neubert, A. Rostagno, and J. Ghiso. 2015. Sequential amyloid- $\beta$ degradation by the matrix metalloproteases MMP-2 and MMP-9. Journal 
of Biological Chemistry 290(24):15078-15091.

30. Porter, K. N., S. N. Sarkar, D. A. Dakhlallah, M. E. Vannoy, D. D. Quintana, and J. W. Simpkins. 2020. Medroxyprogesterone Acetate Impairs Amyloid Beta Degradation in a Matrix Metalloproteinase-9 Dependent Manner. Frontiers in aging neuroscience 12:92.

31. Eckhard, U., P. F. Huesgen, O. Schilling, C. L. Bellac, G. S. Butler, J. H. Cox, A. Dufour, V. Goebeler, R. Kappelhoff, and U. auf dem Keller. 2016. Active site specificity profiling of the matrix metalloproteinase family: Proteomic identification of 4300 cleavage sites by nine MMPs explored with structural and synthetic peptide cleavage analyses. Matrix Biology 49:37-60.

32. Ratnikov, B. I., P. Cieplak, K. Gramatikoff, J. Pierce, A. Eroshkin, Y. Igarashi, M. Kazanov, Q. Sun, A. Godzik, and A. Osterman. 2014. Basis for substrate recognition and distinction by matrix metalloproteinases. Proceedings of the National Academy of Sciences 111(40):E4148-E4155.

33. Overall, C. M. 2002. Molecular determinants of metalloproteinase substrate specificity. Molecular biotechnology 22(1):51-86.

34. Kumar, L., A. Nash, C. Harms, J. Planas-Iglesias, D. Wright, J. Klein-Seetharaman, and S. K. Sarkar. 2020. Allosteric Communications between Domains Modulate the Activity of Matrix Metalloprotease1. Biophysical Journal 119(2):360-374.

35. Kumar, L., J. Planas-Iglesias, C. Harms, S. Kamboj, D. Wright, J. Klein-Seetharaman, and S. K. Sarkar. 2020. Activity-dependent interdomain dynamics of matrix metalloprotease-1 on fibrin. Scientific Reports 10(1):1-14.

36. Wertheim, G., M. Butler, K. West, and D. Buchanan. 1974. Determination of the Gaussian and Lorentzian content of experimental line shapes. Review of Scientific Instruments 45(11):1369-1371.

37. Kozakov, D., D. R. Hall, B. Xia, K. A. Porter, D. Padhorny, C. Yueh, D. Beglov, and S. Vajda. 2017. The ClusPro web server for protein-protein docking. Nature protocols 12(2):255.

38. Comeau, S. R., D. W. Gatchell, S. Vajda, and C. J. Camacho. 2004. ClusPro: a fully automated algorithm for protein-protein docking. Nucleic acids research 32(suppl_2):W96-W99.

39. Yanamala, N., K. C. Tirupula, and J. Klein-Seetharaman. 2008. Preferential binding of allosteric modulators to active and inactive conformational states of metabotropic glutamate receptors. In BMC bioinformatics. BioMed Central. S16.

40. Kumar, L., W. Colomb, J. Czerski, C. R. Cox, and S. K. Sarkar. 2018. Efficient protease based purification of recombinant matrix metalloprotease- 1 in E. coli. Protein expression and purification 148:59-67.

41. Kamboj, S., C. Harms, L. Kumar, D. Creamer, C. West, J. Klein-Seetharaman, and S. K. Sarkar. 2021. A method of purifying alpha-synuclein in E. coli without chromatography. Heliyon 7(1):e05874.

42. Zhuang, X., L. E. Bartley, H. P. Babcock, R. Russell, T. Ha, D. Herschlag, and S. Chu. 2000. A singlemolecule study of RNA catalysis and folding. Science 288(5473):2048-2051.

43. Czerski, J., W. Colomb, F. Cannataro, and S. Sarkar. 2018. Spectroscopic identification of individual fluorophores using photoluminescence excitation spectra. Journal of microscopy 270(3):261-271. 
44. Colomb, W., J. Czerski, J. Sau, and S. Sarkar. 2017. Estimation of microscope drift using fluorescent nanodiamonds as fiducial markers. Journal of microscopy 266(3):298-306.

45. Dittmore, A., J. Silver, S. K. Sarkar, B. Marmer, G. I. Goldberg, and K. C. Neuman. 2016. Internal strain drives spontaneous periodic buckling in collagen and regulates remodeling. Proceedings of the National Academy of Sciences:201523228.

46. Sarkar, S. K., B. Marmer, G. Goldberg, and K. C. Neuman. 2012. Single-molecule tracking of collagenase on native type I collagen fibrils reveals degradation mechanism. Current biology : CB 22(12):1047-1056.

47. Manka, S. W., F. Carafoli, R. Visse, D. Bihan, N. Raynal, R. W. Farndale, G. Murphy, J. J. Enghild, E. Hohenester, and H. Nagase. 2012. Structural insights into triple-helical collagen cleavage by matrix metalloproteinase 1. Proc Natl Acad Sci U S A 109(31):12461-12466.

48. Crescenzi, O., S. Tomaselli, R. Guerrini, S. Salvadori, A. M. D'Ursi, P. A. Temussi, and D. Picone. 2002. Solution structure of the Alzheimer amyloid $\beta$-peptide (1-42) in an apolar microenvironment: Similarity with a virus fusion domain. European Journal of Biochemistry 269(22):5642-5648.

49. Pettersen, E. F., T. D. Goddard, C. C. Huang, G. S. Couch, D. M. Greenblatt, E. C. Meng, and T. E. Ferrin. 2004. UCSF Chimera-a visualization system for exploratory research and analysis. Journal of computational chemistry 25(13):1605-1612.

50. Abraham, M. J., T. Murtola, R. Schulz, S. Páll, J. C. Smith, B. Hess, and E. Lindahl. 2015. GROMACS: High performance molecular simulations through multi-level parallelism from laptops to supercomputers. SoftwareX 1:19-25.

51. Lemkul, J. 2018. From proteins to perturbed Hamiltonians: a suite of tutorials for the GROMACS2018 molecular simulation package [Article v1. 0]. Living journal of computational molecular science 1(1):5068.

52. Wang, J., P. Cieplak, and P. A. Kollman. 2000. How well does a restrained electrostatic potential (RESP) model perform in calculating conformational energies of organic and biological molecules? Journal of computational chemistry 21(12):1049-1074.

53. Mark, P., and L. Nilsson. 2001. Structure and dynamics of the TIP3P, SPC, and SPC/E water models at 298 K. The Journal of Physical Chemistry A 105(43):9954-9960.

54. Arnold, B. C. 2014. Pareto distribution. Wiley StatsRef: Statistics Reference Online:1-10.

\section{Figures}



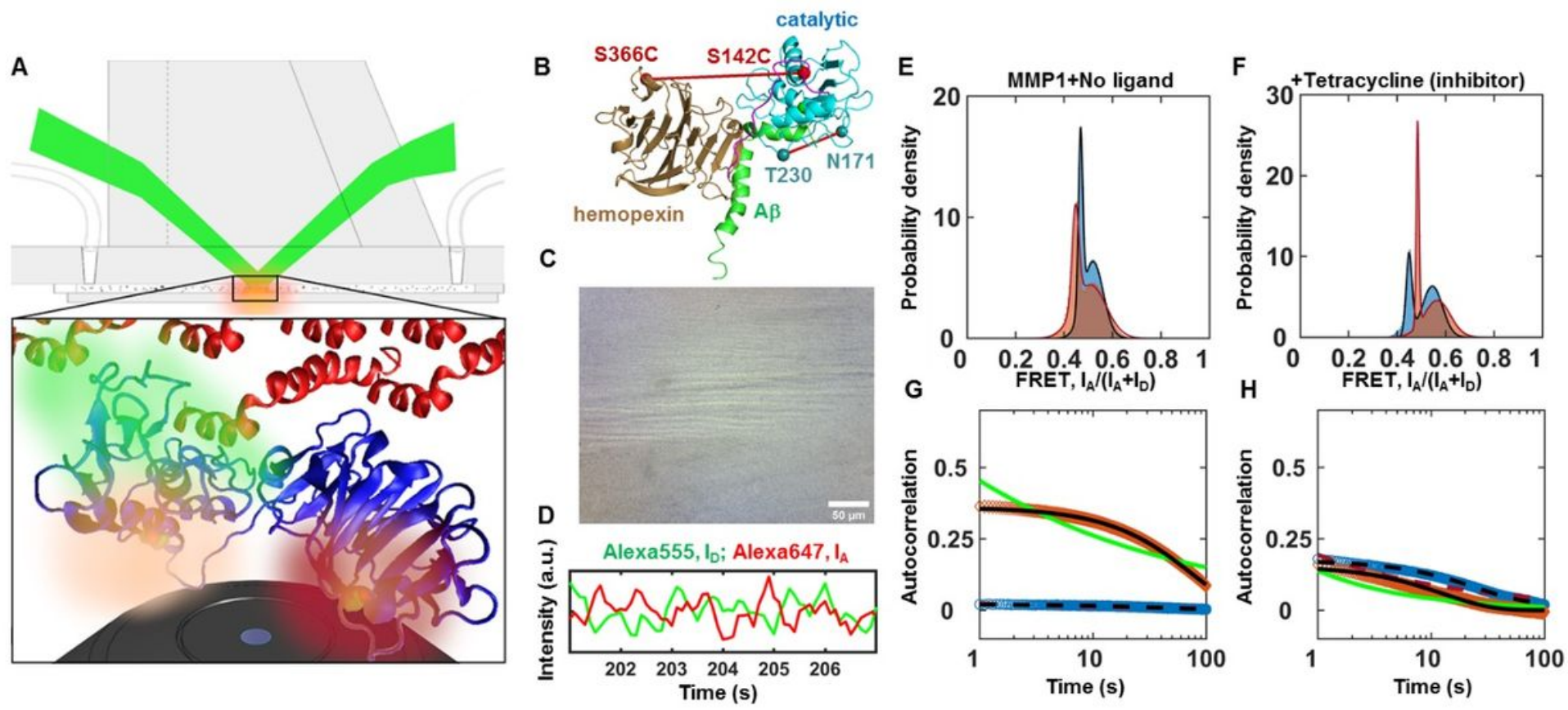

Figure 1

Interdomain dynamics of MMP1 on A 3 -induced aggregates at $22^{\circ} \mathrm{C}$ with $100 \mathrm{~ms}$ time resolution. (A) Schematics of smFRET measurements using a TIRF microscope. (B) One of many binding poses between MMP1 and A 3 . (C) Light microscopy image of $A \beta$-induced aggregates on a slide. (D) Emission intensities of Alexa555 and Alexa647 attached to active MMP1. (E) and (F) Area-normalized histograms of FRET values $(200,000$ FRET values, bin size $=0.005)$ without ligand and in the presence of tetracycline (an inhibitor), respectively, for active (blue) and inactive (orange) MMP1. All histograms are fitted to a sum of two Gaussians (active: solid black line; inactive: solid red line). (G) and $(\mathbf{H})$ Normalized autocorrelations of FRET values without ligand and in the presence of tetracycline, respectively, for active (blue) and inactive (orange) MMP1. All autocorrelations are fitted to exponentials and power laws (exponential fit to active: dashed black line; power-law fit to active: dashed red line; exponential fit to inactive: solid black line; power-law fit to inactive: solid green line). The error bars in histograms and autocorrelations represent the square roots of the bin counts and the standard errors of the mean (SEM) and are too small to be seen. For best-fit parameters, see Table $\mathbf{S 1 .}$ 

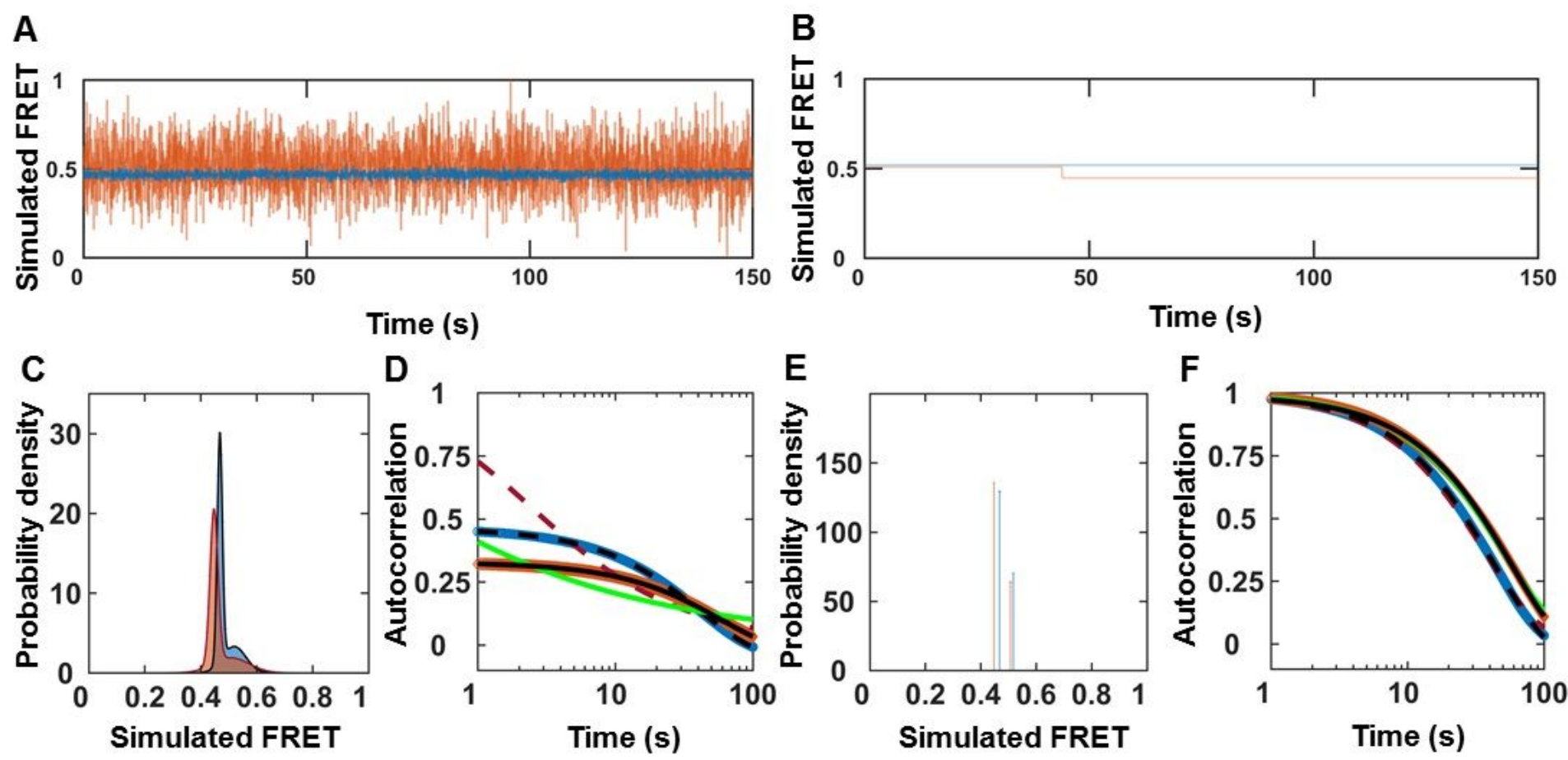

Figure 2

Stochastic simulation of MMP1 dynamics on A $\boldsymbol{\beta}$ as a two-state system. (A) and (B) Examples of simulated smFRET trajectories with and without noise, respectively, for active MMP1 (blue) and inactive MMP1 (orange) using experimental parameters for MMP1 without ligands. (C) Area-normalized histograms of simulated FRET values with noise (active: blue; inactive: orange) with best fits to a sum of two Gaussians (solid black line). (D) Autocorrelations of simulated FRET values with noise (active: blue; inactive: orange) with best fits to exponentials (active: dashed black line; inactive: solid black line). Power law does not fit autocorrelations (active: dashed red line; inactive: solid green line). (E) Area-normalized histograms of simulated FRET values without noise (active: blue; inactive: orange). (F) Autocorrelations of simulated FRET values without noise (active: blue; inactive: orange) with best fits to exponentials (active: dashed black line; inactive: solid black line). Both exponential and power law fit autocorrelations (active: dashed red line; inactive: solid green line). The error bars represent the standard errors of the means (SEM) for histograms and autocorrelations and are too small to be seen. For best-fit parameters, see Table $\mathbf{S 2}$. 
A Pose 1
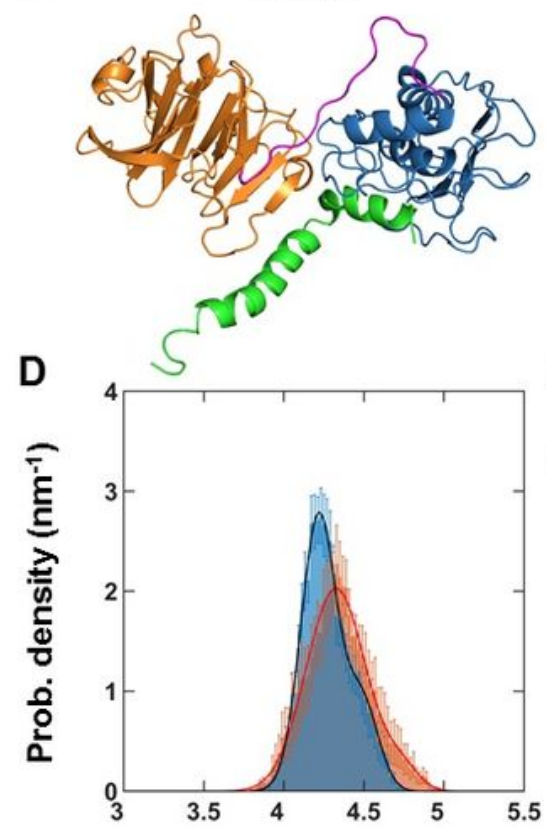

G
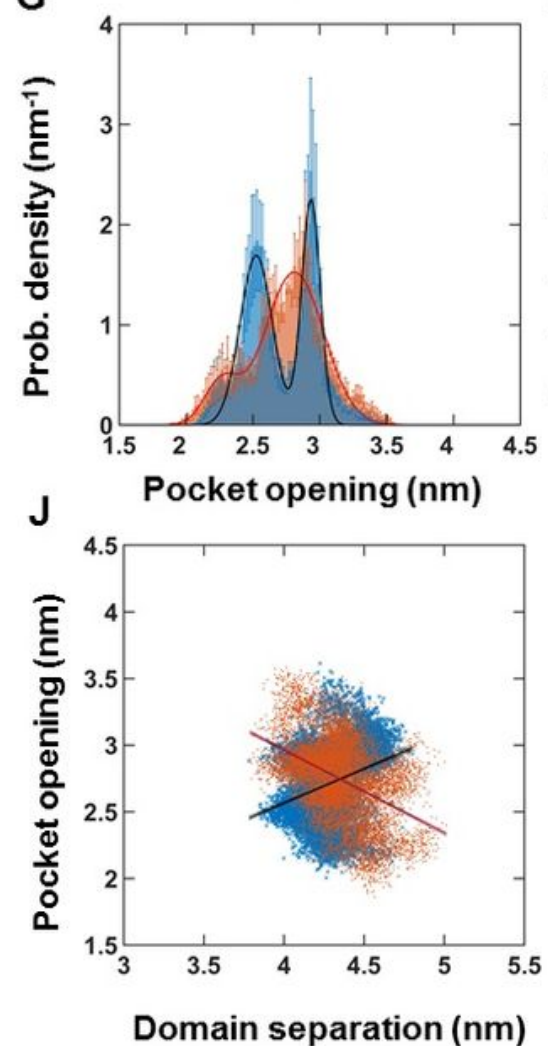

B
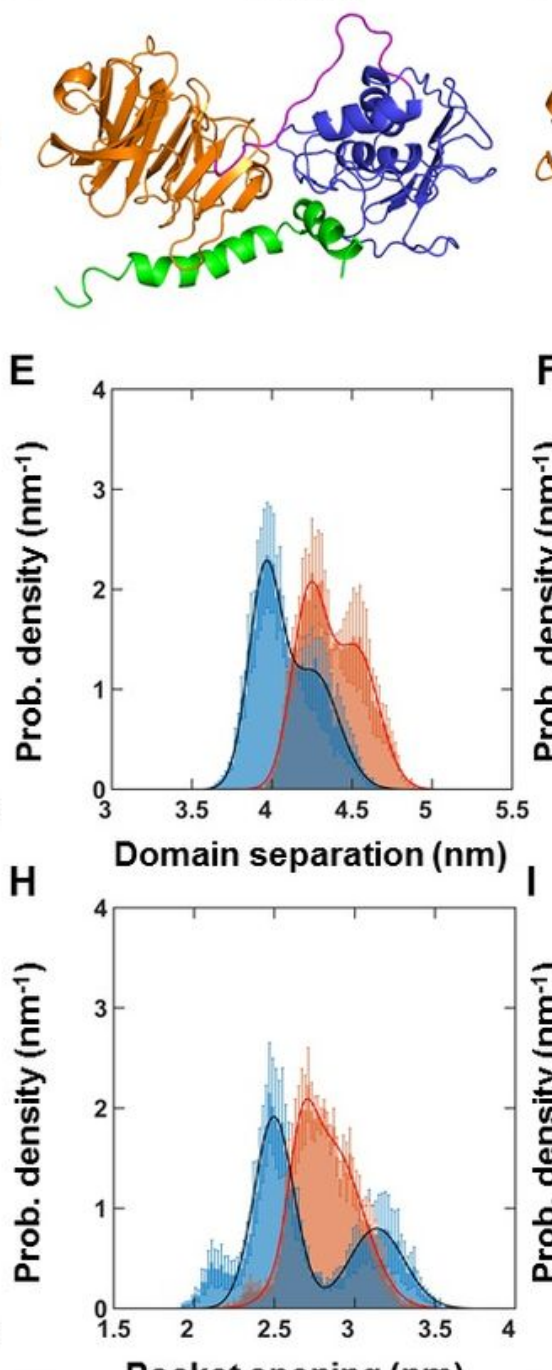

$\mathrm{K}$
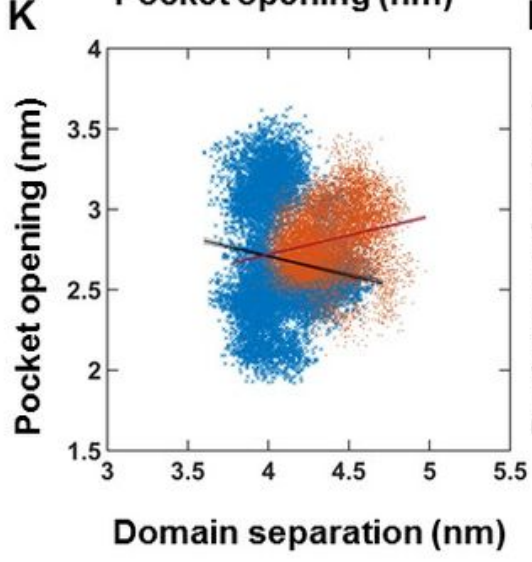

C Pose 3
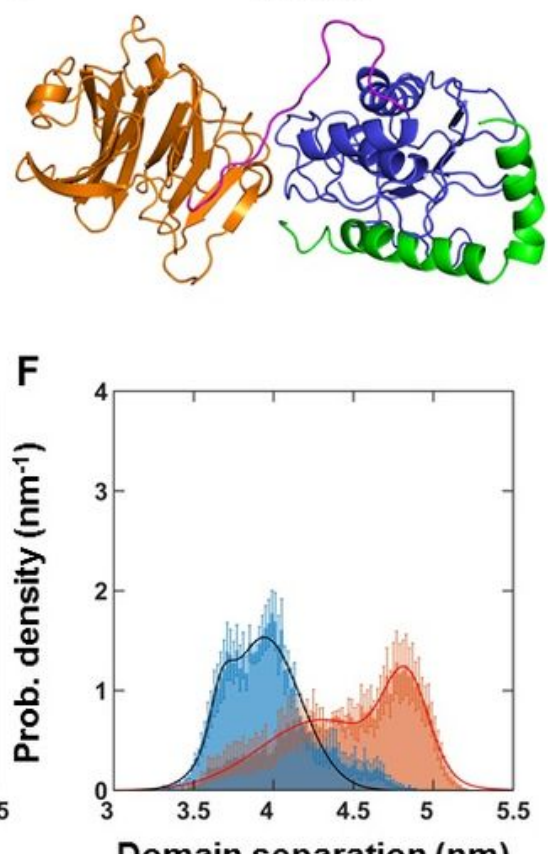

Domain separation (nm)

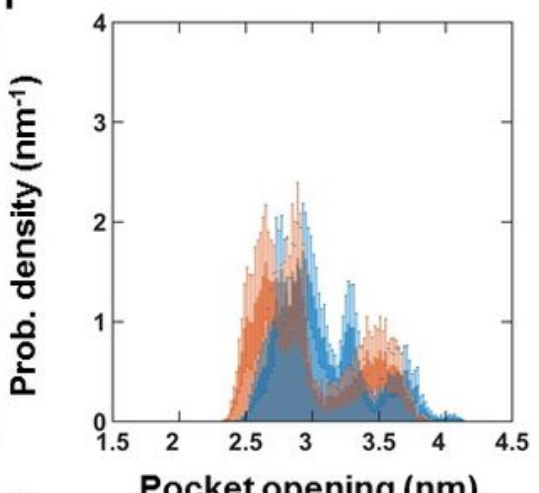

$\mathbf{L}$

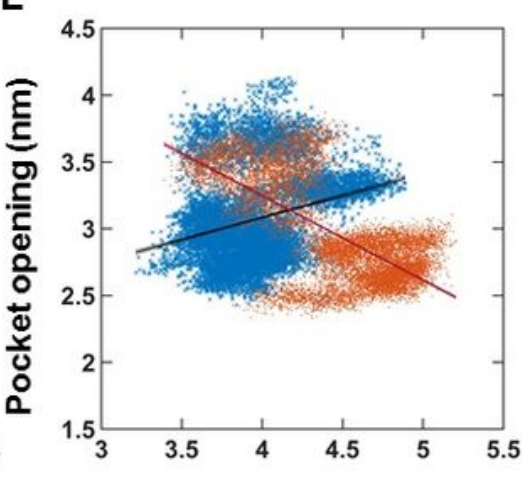

Domain separation $(\mathrm{nm})$

\section{Figure 3}

MMP1-A $\beta$ binding pose-dependent dynamics of MMP1 at $22^{\circ} \mathrm{C}$. (A-C) Three top-scoring binding poses of MMP1 (catalytic domain in blue and $A \beta$ in green) with $A \beta$ (PDB ID 1IYT) predicted by ClusPro. (D-F) Areanormalized histograms (active MMP1: blue; inactive MMP1: orange) of interdomain separation of MMP1 (S142-S366 distance) for poses 1, 2, and 3, respectively. All histograms are fitted to a sum of two Gaussians (active MMP1: solid black line; inactive MMP1: solid red line). (G-I) Area-normalized 
histograms (active MMP1: blue; inactive MMP1: orange) of the catalytic pocket opening (N171-T230 distance) of MMP1 for poses 1, 2, and 3, respectively. (J-L) Linear correlation plots of the catalytic pocket opening and interdomain distance (active MMP1: blue; inactive MMP1: orange) for poses 1, 2, and 3, respectively. The data were fitted to $y_{i}=b_{0}+b_{1} \times x_{i}$ (Equation 4 in methods). Note that a larger domain separation corresponds to a lower FRET value. Time resolution $=2 \mathrm{fs}$; Data saved every $5 \mathrm{ps}$; RMSD stabilization time for MMP1 $=\sim 5 \mathrm{~ns}$; Total simulation duration=20 ns. For calculations of linear correlations, see methods. For best-fit parameters, see Table S3. The error bars in Figures 3D-I represent the standard deviations of three repeats of simulations for each condition. Figures $3 \mathrm{~J}-\mathrm{L}$ show combined data from the three repeats.
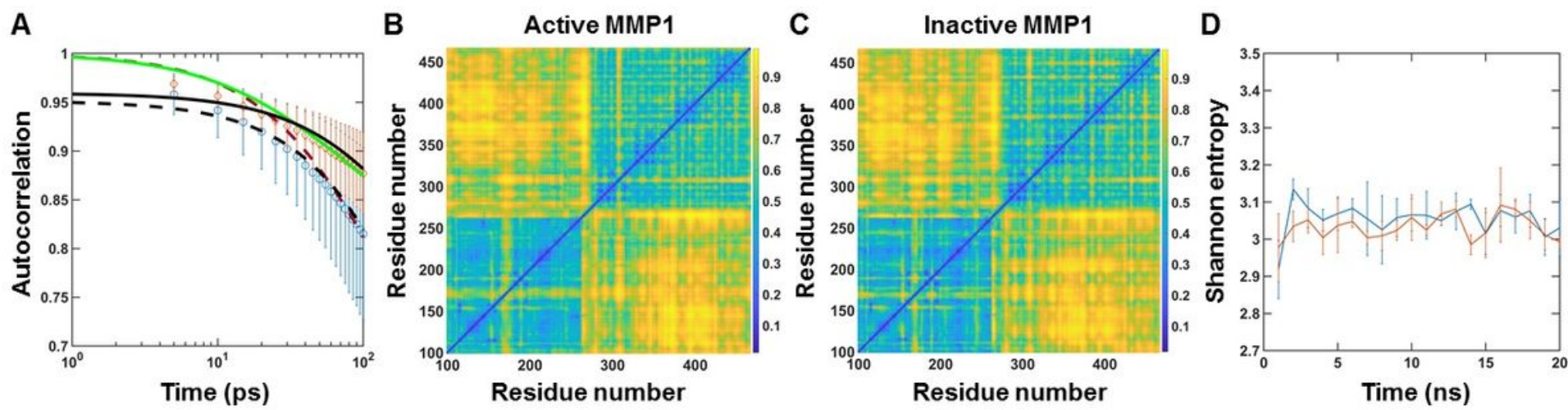

Figure 4

Quantification of MMP1 allostery on A $\beta$ at $22^{\circ} \mathrm{C}$ for pose 3. (A) Autocorrelations of simulated interdomain distance (active: blue; inactive: orange) with best fits to exponentials (active: dashed black line; inactive: solid black line) and power law (active: dashed red line; inactive: solid green line). (B) and (C) Correlations of position fluctuations between every pair of residues for active and inactive MMP1, respectively. (D) Shannon entropy calculated from correlation plots for active ( $S=3.06 \pm 0.04$, mean $\pm S E M)$ and inactive $(S=3.03 \pm 0.03$, mean $\pm S E M)$. For best-fit parameters, see Table $\mathbf{S 4}$.
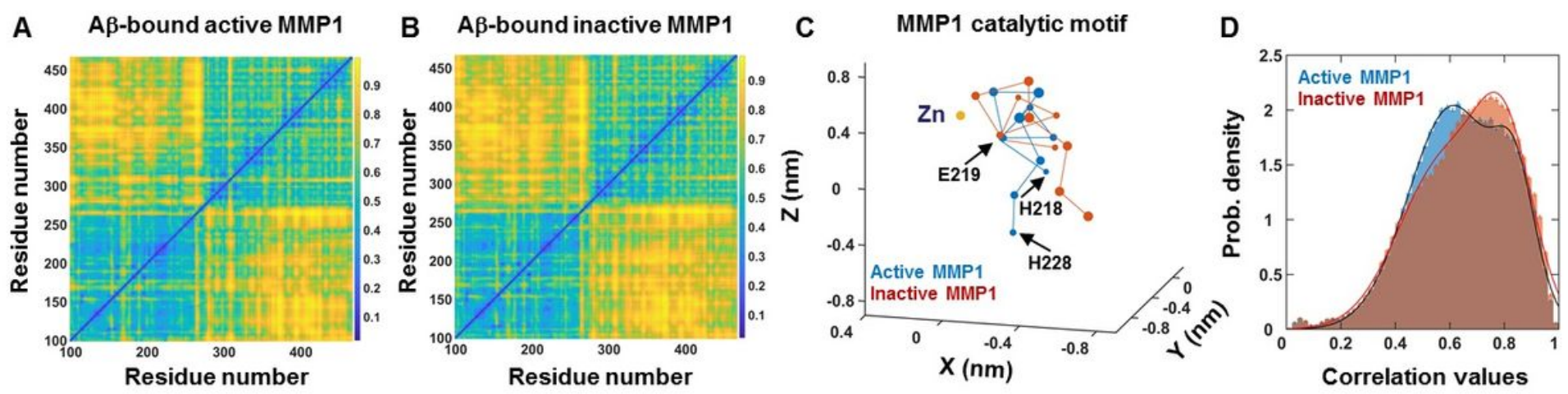

Figure 5 
(B) Normalized correlations between each pair of residue for A $\beta$-bound inactive MMP1. (C) Threedimensional configurations of the catalytic motif residues for $A \beta$-bound active MMP1 (blue) and A bound inactive MMP1 (red). (D) Histograms of correlation values for A $\beta$-bound active MMP1 (blue) and A $\beta$-bound inactive MMP1 (red).

\section{A A $\beta$-bound MMP1 Pose 1}

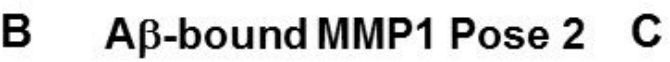

A $\beta$-bound MMP1 Pose 3
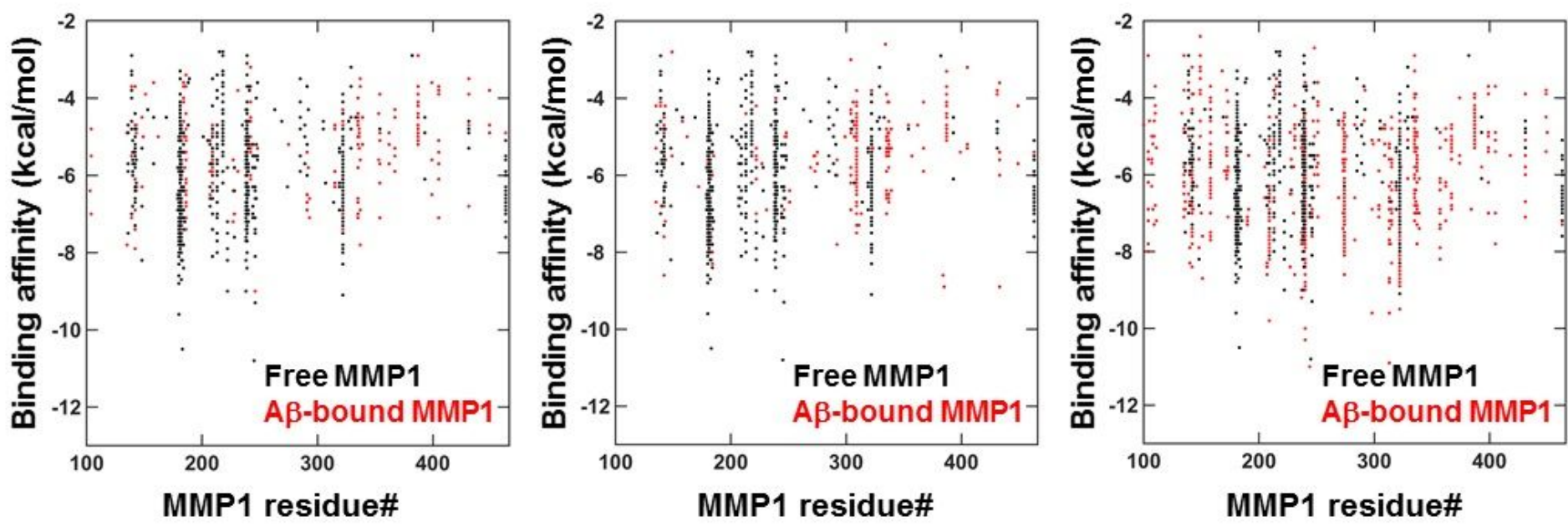

D

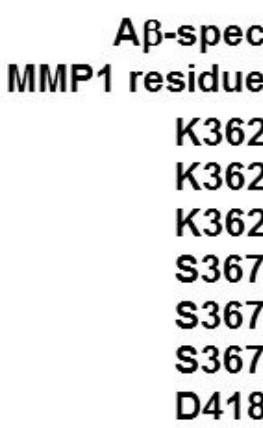

$\begin{array}{lc}\text { Drug } & \begin{array}{c}\text { Affinity } \\ \text { (kcal/mol) }\end{array} \\ \text { Trosec } & -7.2 \\ \text { Bumetanide } & -6.3 \\ \text { Azelex } & -6.3 \\ \text { Zanamivir } & -6.9 \\ \text { Piroxicam } & -6.9 \\ \text { Naltrexone } & -6.8 \\ \text { Cefa } & -5.5\end{array}$

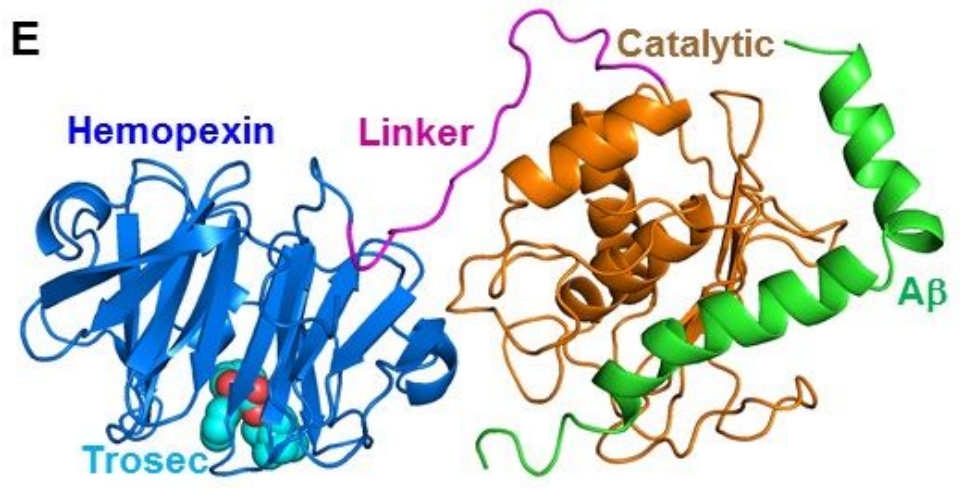

Figure 6

Substrate- and posedependent virtual screening of molecules against MMP1. Binding affinities of lead compounds and closest MMP1 residues for A -bound MMP1 in (A) pose 1, (B) pose 2, and (C) pose 3. (D) Compounds that bind to A $\beta$-specific allosteric residues in MMP1 in pose 3. (E) Trosec bound to Ab-bound MMP1 in pose 3. Lead molecules depend on whether MMP1 is free or bound to A $\beta$. Also, not all identified allosteric residues have associated lead molecules. Allosteric residues reduce the number of lead molecules significantly.

\section{Supplementary Files}

This is a list of supplementary files associated with this preprint. Click to download.

- ABMMP1InteractionsSI01132022.pdf 